Relations industrielles

Industrial Relations

\title{
L'Acte des manufactures de Québec, 1885: un centenaire
}

\section{André C. Côté}

Volume 40, numéro 3, 1985

URI : https://id.erudit.org/iderudit/050163ar

DOI : https://doi.org/10.7202/050163ar

Aller au sommaire du numéro

Éditeur(s)

Département des relations industrielles de l'Université Laval

ISSN

0034-379X (imprimé)

1703-8138 (numérique)

Découvrir la revue

Citer cet article

Côté, A. C. (1985). L'Acte des manufactures de Québec, 1885: un centenaire. Relations industrielles / Industrial Relations, 40(3), 623-628.

https://doi.org/10.7202/050163ar
Résumé de l'article

L'acte des manufactures de Quebec, 1885 : un centenaire
Tous droits réservés @ C Département des relations industrielles de l'Université Laval, 1985
Ce document est protégé par la loi sur le droit d'auteur. L’utilisation des services d'Érudit (y compris la reproduction) est assujettie à sa politique d'utilisation que vous pouvez consulter en ligne.

https://apropos.erudit.org/fr/usagers/politique-dutilisation/ 


\title{
L'Acte des manufactures de Québec, 1885 Un centenaire
}

\author{
André C. Côté
}

Il y a précisément cent ans, le 9 mai 1885 , était sanctionnée la première grande loi québécoise du travail, l'Acte pour protéger la vie et la santé des personnes employées dans les manufactures, autrement désignée sous son titre abrégé comme l'Acte des manufactures de Québec, 1885'.

Le texte de cette loi s'inspirait largement d'une loi ontarienne adoptée l'année précédente ${ }^{2}$, laquelle tirait son inspiration des lois britanniques antérieures de même nature, en particulier du Factory and Workshop Act de $1878^{3}$.

Alors que la compréhension des paramètres du partage des compétences constitutionnelles respectives du fédéral et des provinces était encore à cet égard imprécise, le Parlement d'Ottawa, après avoir tergiversé sur divers projets de loi analogues au début des années $1880^{4}$, avait créé une commission d'enquête sur la condition des ouvriers des manufactures ${ }^{5}$.

* CÔTÉ, André C., professeur, Faculté de droit, Université Laval.

1 (1885) 48 Vict. L.Q., c. 32.

2 Act for the Protection of Persons Employed in Factories, (1884) 47 Vict. S.O., c. 39.

3 (1878) 41-42 Vict., c. 16. Cette loi refondait diverses lois antérieures dont la première était la Health and Morals of Appendices Act, (1802) 42 Geo III, c. 73. Voir à cet égard E.L. HUTCHINS et A. HARRISSON, A History of Factory Legislation, 3rd ed., A.M. Kelly, New York, 1966.

4 Le 2 mai 1879, le «Bill pour régler le travail des enfants et des jeunes personnes dans les moulins et manufactures du Canada» était présenté en première lecture. Le 19 février 1880 , ce projet de loi était remplacé par le «Bill pour régler le travail des enfants, des jeunes personnes et des femmes dans les ateliers et fabriques du Canada», également présenté en première lecture. Le 17 décembre 1880 , ce projet de loi était à nouveau remplacé par le «Bill tendant à régler les heures de travail dans les ateliers, moulins et fabriques du Canada et pour autres fins». Le 23 février 1881, ce projet de loi était retiré à l'occasion de sa deuxième lecture, du consentement de la Chambre, sous la foi d'une promesse du gouvernement de désigner une commission royale d'enquête sur la condition des ouvriers.

5 Voir à cet égard, R. DESROSIERS et D. HÉROUX, Le travailleur québécois et le syndicalisme, P.U.Q., 1973, pp. 87-96; également, A.E. GRAUER, Législation ouvrière: étude préparée pour la Commission royale des relations entre le Dominion et les provinces, Ottawa, 1939. 
Le «Rapport des commissaires chargés de faire une enquête sur le fonctionnement des moulins et fabriques du Canada, et sur la main-d'oeuvre qui est employée», remis au gouvernement fédéral le 18 janvier 1882, décrivait, en particulier, l'exploitation du travail des enfants et les conditions de travail souvent dangereuses, insalubres et sordides des ouvriers ${ }^{6}$. Ce document n'est sans doute pas étranger à l'adoption de ces lois par l'Ontario et le Qué$\mathrm{bec}^{7}$. Incidemment et de façon implicite, ces provinces affirmaient ainsi, face aux hésitations du gouvernement central, leur compétence constitutionnelle en la matière.

La loi de 1885 visait d'une part à «protéger la vie et la santé des personnes employées dans les manufactures», par la fixation de certaines balises destinées à assurer un minimum de salubrité et de sécurité dans les manufactures. D'autre part, le législateur s'y attaquait à l'exploitation du travail des femmes et des enfants.

Pour assurer son application, son article 14 permettait la constitution d'un corps d'inspecteurs investis de pouvoirs d'enquête et seuls habilités à intenter les poursuites en vertu de cette loi qui ne devait pas être considérée, selon les termes de son article 37, comme modifiant «les dispositions des lois civiles de cette province concernant la responsabilité du patron envers son employé».

En plus des amendes, ces inspecteurs pouvaient requérir des tribunaux des ordonnances «que certains moyens soient adoptés par le patron dans les délais fixés par cette ordre (sic), pour mettre sa manufacture en règle avec les dispositions du présent acte» (art. 27).

Au plan substantif, cette loi comprenait une première section intitulée «Mode de tenir les manufactures». L'article 3 proclamait qu' «il n'est pas permis de tenir une manufacture de manière que la vie de qui que ce soit qui y est employé soit en danger, ou de façon que la santé de ceux qui y sont employés soit probablement en danger d'être permanemment compromise».

Plus précisément, cette section contenait des dispositions relatives à la propreté et à l'aération des lieux de travail, à l'expulsion par moyen mécanique des poussières dangereuses et à la disponibilité de lieux d'aisance adéquats et séparés pour l'usage de chaque sexe (arts 4 et 5).

On retrouve également dans cette section des stipulations relatives à l'installation d'appareils protecteurs pour entourer les équipements mobiles ou dangereux, ainsi que pour assurer la sécurité des ascenseurs et autres appareils de levage (art. 7). Enfin, la loi prévoyait l'installation d'issues de sauvetage et d'autres moyens de prévention des incendies (art. 8).

6 Rapport des commissaires chargés de faire une enquête sur le fonctionnement des moulins et fabriques du Canada, et sur la main-d'oeuvre qui est employée, (1882) 45 Vict., Documents de la session, no 42.

7 Lors de la présentation de projets de loi en deuxième lecture, le 22 mars 1885 , le procureur-général Taillon affirmait ceci: «M. le Président, dans l'élaboration de ce projet de loi, je dois dire que j'ai profité de ce qui a été fait à Ottawa et à Ontario (sic)", Journal des débats de l'Assemblée législative de Québec, $4^{\mathrm{e}}$ session de la $5^{\mathrm{e}}$ législature, 1885, p. 318. 
Pour comprendre l'importance que revêtait ce nouveau texte de loi, il faut lire les rapports et témoignages de ses contemporains qui font état de conditions d'hygiène et de sécurité, parfois invraisemblables à nos yeux, règnant dans certaines fabriques: ateliers situés dans des sous-sols humides et mal éclairés, machines dangereuses sans aucune protection, machines à vapeur et chaudières placées sous la surveillance d'enfants ou de personnes inexpérimentées, chaudières situées dans les lieux de travail, etc. ${ }^{8}$.

On est particulièrement frappé par l'ignorance, l'indifférence et le cynisme qui régnaient parfois. À titre d'exemples, certains employeurs s'objectaient à l'installation d'escaliers de secours en prétextant les dangers de vols $^{9}$. Que dire, par ailleurs, de l'atmosphère qui devait régner dans certains lieux de travail surpeuplés et encombrés, sans facilités sanitaires minimalement décentes et dont les seules sources de ventilation étaient les portes et les fenêtres qui devaient rester fermées l'hiver, à cause du froid! ${ }^{10}$

L'exploitation abjecte, par ailleurs, du travail de jeunes enfants était une situation répandue qui allait même, aux dires des Commissaires de 1882 en s'accentuant, la demande étant plus forte que l'offre ${ }^{11}$. Les Commissaires recensaient, parmi les quelque 43511 ouvriers des usines considérées, 104 garçons et 64 filles de moins de dix ans et 1263 garçons et 823 filles de 10 à 14 ans.

Le rapport de 1882 est fort révélateur de certaines mentalités de l'époque. On y rationnalisait ainsi l'emploi des jeunes enfants en usine, nous disent les Commissaires:

«Raisons pour lesquelles on emploie de jeunes enfants:

1) que le travail est facile;

2) que de plus âgés ne le pourraient faire;

3) que les fabricants rivaux emploient cette main-d'oeuvre;

4) comme il n'y a pas de loi ou de défense à ce sujet, quelques-uns se servent de cette main-d'oeuvre et ceux qui ont des idées plus libérales sont obligés de faire de même.»13

La loi de 1885 intervenait donc pour la première fois dans la sacrosainte liberté contractuelle en prohibant, dans sa seconde section, le travail des enfants de sexe masculin âgés de moins de 12 ans et ceux de sexe féminin âgés de moins de 14 ans, dans les manufactures (art. 9).

Quant aux heures de travail, une étude faite pour la Commission royale d'enquête fédérale sur les relations entre le travail et le capital devait révéler ceci:

8 En plus du rapport de 1882, supra note 6, la source de renseignements la plus riche se retrouve au Report of the Royal Commission on the Relations of Labor and Capital in Canada, Imprimeur de la Reine, Ottawa, 1889, 3 vol., dont les annexes recèlent de témoignages bouleversants. Voir également, J. BERNIER, «La condition des travailleurs, 1851-1896», in J. Hamelin (éd.), Les travailleurs québécois 1851-1896, P.U.Q., 1973, pp. 31-55.

9 Loc. cit., supra, note 6, p. 7.

10 Id., p. 8.

11 Id., p. 2.

$12 I d .$, p. 11. 
«(...) in the Province of Quebec much evidence of long-continued labor came before the Commission. This is to be the more deplored in cases where children, especially very young children, are employed. At some cotton mills, in which children not exceeding nine years of age are employed, the work is frequently continuous from 6:30 in the morning till noon, and from 12:45 till 7:30 in the afternoon, making thirteen hours of work, with only one intermission of three-quarters of an hour, and having an uninterrupted stretch of nearly seven hours. On rare occasions the machinery is kept in operation from 12:45 p.m. till 9 o'clock, without stopping although some opportunity is given the operatives to snatch a bite of supper. In nearly all cities throughout the Dominion conductors and drivers on street railway cars are required to work very long hours. Some of them are employed from 6 o'clock in the morning till 10 o'clock at night, though they are actually on the cars not more than twelve hours in a day. The best retail shops of dry goods merchants in all cities are open only from 8 o'clock a.m. till 6 o'clock p.m.; but in many others the hours are very long, both for clerks and for other employees. At some shops in Montreal the clerks are employed from 5:30 a.m. till 10 or even 11 o'clock at night. Dressmakers and milliners are kept, during busy seasons, till even later hours. In October, November and December they are, in some places, kept at work from 8 in the morning till midnight, and on Saturday nights till far into the hours of Sunday morning. Children in the millinery rooms are at work from 6 in the morning till 9 at night, with brief intervals for meals. $(\ldots){ }^{13}$

Intervenant timidement pour des raisons philanthropiques et humanitaires, et au seul bénéfice de ceux qui étaient perçus alors comme les plus faibles et les plus démunis, le législateur, dans la troisième section de la loi de 1885 , limitait en principe à dix heures par jour et soixante heures par semaine l'horaire de travail effectif des enfants de 12 à 14 ans, ou encore des jeunes filles ou des femmes travaillant dans les manufactures (art. 10).

La loi obligeait l'employeur à accorder aux enfants, jeunes filles ou femmes employés dans les manufactures, au moins une heure chaque jour, le midi, pour le repas, tout en stipulant que «cette heure n'est pas comptée comme partie du temps par le présent limité à l'égard de leur travail (sic)». Sans en faire un principe, la loi permettait par ailleurs à l'inspecteur d'ordonner au patron de permettre aux enfants, jeunes filles et femmes de prendre leurs repas dans une pièce autre que leur lieu de travail.

Il est remarquable de constater que ce même article permettait néanmoins d'augmenter la durée de la journée de travail dans le but d'abréger la journée de travail du samedi. À cet égard, les observations du rapport de 1882 sont dignes de mention:

"Quelques maîtres, y lit-on, sont fortement opposés aux demi-congés du samedi, tandis que les serviteurs les désirent bien certainement. Les premiers s'y opposent sur le principe que cela diminue leur commerce ou la quantité des objets qu'ils fabriquent et que les ouvriers perdront ce temps en débauches. Que cela nuise au commerce, il y a tout lieu de le croire, mais les Commissaires ne pensent pas que ces congés auront le mauvais effet dont il est parlé; au contraire, leurs observations et leur expérience les ont convaincus que les artisans et les ouvriers du Canada étaient aussi intelligents, industrieux et sobres que ceux de tout autre pays. $\rangle^{14}$

$13 \quad I d .$, p. 3.

14 Loc. cit., supra, note 8, annexe F, p. 37. 
Pour en revenir à la loi de 1885 , disons enfin que, dans des circonstances particulières, pour rattraper le temps perdu suite à un accident ou à un bris d'équipement, ou encore "pour satisfaire au besoin et aux exigences de l'exploitation industrielle», celle-ci permettait d'habiliter par règlement l'inspecteur à exempter un patron des limitations imposées par la loi, ce, dans les limites suivantes:

"pourvu, toutefois, (...) qu'aucun enfant, aucune jeune fille ou femme, ne soit employé avant six heures du matin ni après neuf heures du soir, et que la durée du travail d'un enfant, d'une jeune fille et d'une femme, ne dépasse pas douze heures et demie de travail par jour, ni soixante et douze heures et demie par semaine, et que l'exemption ne s'étende pas à plus de six semaines en aucune année, et que le temps réservé par le présent acte pour les repas ne soit pas réduit» (art. 13).

Malgré ce que pourrait laisser suggérer la lecture de ces divers témoignages, «la loi de 1885 venait s'insérer dans un contexte social assez mal préparé». Comme le soulignait ainsi Roger Chartier:

«l'enquête fédérale sur la condition ouvrière (de 1882) n'avait ému qu'un petit nombre de personnes éclairées au Québec; le peuple lisait peu, et son conformisme au travail, le manque d'une prise de conscience collective de situations industrielles trop souvent indignes d'êtres humains firent que le cabinet Ross préconisa l'adoption de l'«Acte des manufactures» sans qu'il se produisit de remous dans l'opinion publique. C'était vraiment, ici, la loi précédant les moeurs». ${ }^{16}$

Ce n'est que trois ans jour pour jour après la sanction de la loi, le 9 mai 1888, qu'étaient nommés les trois premiers inspecteurs des manufactures, Charles T. Côté, James Mitchell et Louis Guyon. Le fait que la loi de 1885 ait été ainsi enfin activée découlait, semble-t-il, de l'intérêt soulevé par les audiences et les travaux de la Commission royale d'enquête fédérale sur les relations entre le travail et le capital, nommée en $1886^{17}$.

Fait cocasse, ces premiers inspecteurs relevaient du ministre de l'Agriculture et de la Colonisation. On en était encore loin de la création d'un ministère du Travail qui ne devait intervenir qu'en $1931^{18}$.

Les rapports annuels de ces premiers inspecteurs ${ }^{19}$ devaient avoir une influence marquante sur l'évolution de cette loi qui était amendée dès 1890 pour être ensuite remplacée par la Loi des établissements industriels de Québec ${ }^{20}$ de 1894. Celle-ci allait devenir la Loi des établissements industriels

15 Loc. cit., supra, note 6, p. 5.

16 R. CHARTIER, «L'inspection des établissements industriels et des édifices publics (1885-1900)", Relations industrielles, vol. 17, no 1, 1962,pp. 43-58.

17 Id., p. 47.

18 Loi concernant le département du travail, L.R.Q. 1925, c. 95A, ajouté par la Loi modifiant les lois concernant l'organisation des départements, 1931, L.Q., c. 19, art. 6. Auparavant, la responsabilité des inspecteurs des manufactures était passée au ministre des travaux publics.

19 On retrouve ces rapports annuels des inspecteurs des manufactures à compter de 1889 dans les Rapports du Commissaire de l'agriculture et de la Colonisation de la Province de Québec, Imprimeur de la Reine, Québec, publiés annuellement.

20 (1894) 57 Vict. L.Q., c. 30. 
et commerciaux ${ }^{21}$. Sans changement d'approche ou de philosophie d'intervention, cette loi perpétuait l'oeuvre amorcée par la loi de 1885 , ce, jusqu'à son remplacement par l'entrée en vigueur de la Loi sur la santé et la sécurité du travail en $1979^{22}$.

En application des principes affirmés à son article 2, cette loi nouvelle substitue une approche participative à la philosophie plutôt répressive qui caractérisait les lois antérieures. D'autre part, elle proclame le principe de «l'élimination à la source même des dangers pour la santé, la sécurité et l'intégrité physique des travailleurs». Enfin et surtout, la loi de 1979, au lieu de ne s'adresser qu'au patron en sa qualité de propriétaire de l'établissement, intervient au niveau même de la relation de travail en articulant un régime de droits et obligations des employeurs et des salariés en matière de santé et de sécurité au travail.

\section{LA SYNDICALISATION DANS LE SECTEUR PRIVÉ AU QUÉBEC}

Préface: Gilles FERLAND - Introduction: Jacques BELANGER, Jean BOIVIN, Claude RONDEAU, Jean SEXTON - Le syndicalisme dans l'entreprise: tendances récentes et analyse, Claude RONDEAU et Jacques BELLANGER - La syndicalisation dans le contexte économique québécois, Sidney INGERMAN - Commentaires: Bernard BONIN - L'impact du régime de relations du travail sur la syndicalisation, Gilles LAFLAMME - Commentaires: Hélène LEBEL - Les idées de réforme sur la syndicalisation au Québec depuis 1964, Jacques DESMARAIS - Table ronde: Organisation syndicale: difficultés et motifs de résistance, Pierre MERCILLE, Bertin NADEAU, Madeleine OLIVIER - Pourquoi et comment accroître la syndicalisation dans le secteur privé? Monique SIMARD, Raymond SLIGER - Commentaires: Gérard DION L'entreprise peut-elle se passer d'un syndicat?, Charles PERREAULT - Commentaires: Fernand GAUTHIER, Paul-Martel ROY - La politique gouvernementale en matière de syndicalisation, Raynald FRECHETTE - Table ronde: Les réactions du milieu, Claire BONENFANT, Ghislain DUFOUR, Jean-Paul HÉTU - Syndicalisme, nouvelles technologies et incertitudes économiques, Guy CAIRE.

ISBN 2-7637-7030-4 1 volume, 276 pages - 1983 - Prix $\$ 17.00$ Les Presses de l’Université Laval

Cité universitaire C.P. 2447, Québec, P.Q., Canada, G1K 7R4

21 (1964) S.R.Q., c. 150 remplacée par la Loi sur la santé et la sécurité du travail, 1979 L.Q., c. 63, art. 285.

22 L.R.Q., c. S-2.1. 